Livraisons

d'Histoire

de l'Architecture

\section{Livraisons de l'histoire de l'architecture}

$17 \mid 2009$

Émotions patrimoniales I

\title{
Léon Parvillée : dialogue entre architecture et arts décoratifs
}

Léon Parvillée: dialogue between architecture and decorative arts

Léon Parvillée : Dialog zwischen Architektur und dekorativer Kunst

\section{Caroline Gronier}

\section{(2) OpenEdition}

\section{Journals}

Édition électronique

URL : http://journals.openedition.org//ha/212

DOI : $10.4000 /$ /ha. 212

ISSN : $1960-5994$

Éditeur

Association Livraisons d'histoire de l'architecture - LHA

Édition imprimée

Date de publication : 10 juin 2009

Pagination : 95-106

ISSN : 1627-4970

Référence électronique

Caroline Gronier, "Léon Parvillée : dialogue entre architecture et arts décoratifs », Livraisons de

I'histoire de l'architecture [En ligne], 17 | 2009, mis en ligne le 10 juin 2011, consulté le 01 avril 2020

URL : http://journals.openedition.org//ha/212 ; DOI : https://doi.org/10.4000//ha.212

Ce document a été généré automatiquement le 1 avril 2020.

Tous droits réservés à l'Association LHA 


\title{
Léon Parvillée : dialogue entre architecture et arts décoratifs
}

\author{
Léon Parvillée: dialogue between architecture and decorative arts \\ Léon Parvillée : Dialog zwischen Architektur und dekorativer Kunst
}

\section{Caroline Gronier}

1 L'éventail des compétences de Léon Parvillée, ainsi que les différentes appellations sous lesquelles il est enregistré aux expositions et dans les almanachs du commerce, expliquent les multiples facettes qu'on prête à sa carrière et à son travail. Or, qu'on le présente comme «sculpteur-ornemaniste», "architecte-décorateur » ou " céramiste orientaliste ", voire "défenseur de la céramique architecturale ", c'est toujours de décor et d'architecture qu'il s'agit dans son œuvre. Sculpteur à ses débuts, il crée les décorations intérieures de nombreux édifices à Istanbul, puis, après plusieurs travaux d'architecte pour le gouvernement ottoman, se lance dans la céramique architecturale et la faïence d'art en France. Ces deux domaines de la céramique alimenteront ses recherches jusqu'à sa mort; dans ces deux domaines, ses réalisations le hisseront parmi les artistes novateurs et les principaux créateurs de son temps. L'art et les monuments turcs qu'il découvre au cours de son long séjour dans l'Empire ottoman lui donnent la marque d'originalité qui le caractérisera à son retour en France. Le livre sur l'art turc qu'il publie en 1874 sous le haut parrainage de Viollet-le-Duc finira de l'asseoir aux côtés des connaisseurs du monde méditerranéen. Mais l'art ottoman lui fait surtout comprendre les possibilités infinies et les qualités multiples de la céramique, en particulier en matière de décoration architecturale. Léon Parvillée décède le 14 août 1885 , après avoir été nommé chevalier de la légion d'honneur.

\section{Sculpteur, décorateur, architecte ou céramiste ? Les différentes facettes de la carrière de Parvillée}

Louis Léon Parvillée nait le 22 mars 1830 à Paris. Fils de menuisier, il obtient à treize ans une bourse de la Ville et entre comme élève à l'« École royale gratuite de dessin »1 
où il accomplit toute sa scolarité jusqu'à ses dix-huit ans. Il y suit les nombreux cours de dessin dans lesquels il excelle et gagne plusieurs prix aux concours de l'École 2 . Il y est également formé aux mathématiques, à la sculpture et à l'architecture, assiste aux cours d'« histoire générale de l'ornement » et de « composition ornementale » d'Eugène Viollet-le-Duc dont la personnalité le marque profondément. Il restera en contact avec son illustre professeur jusqu'à la mort de celui-ci en 1879, lui demandant conseil et se référant à son enseignement à de nombreuses reprises.

\section{Diffuser le goût français en Turquie}

3 Lorsqu'il est recruté pour le service militaire en 1850, Parvillée est sculpteur dans le XI arrondissement de Paris. En 1851, sa carrière prend soudain un tournant décisif. Son ami Pierre-Victor Galland, peintre-décorateur, lui propose, de l'accompagner à Istanbul pour travailler à la décoration intérieure du palais Djézairly dont la construction vient d'être achevée. Mais le propriétaire du palais fait banqueroute, le projet s'arrête deux ans plus tard. Léon Parvillée, installé dans la capitale ottomane et après un bref passage comme ingénieur dans une fonderie de canons, ouvre un atelier de sculpture dans le quartier de Péra en 1854. Dans le cadre de cet atelier, ce sont les techniques décoratives utilisées traditionnellement en Europe qu'il propose. L'annonce publicitaire qu'il fait paraître dans le Journal de Constantinople entre août et novembre de cette même année mentionne :

Parvillée - Sculpteur sur marbre, plâtre, bois, pierre - Ornements en carton-pierre pour bâtiments - Monuments funèbres - Moulages sur nature, etc. Aux quatre rues de Péra.

C'est en « carton-pierre $»^{3}$ d'ailleurs que Parvillée réalise l'une de ses toutes premières commandes en Turquie: le décor de l'église catholique de l'Enfant Jésus, église restaurée par l'architecte officiel de la cour impériale russe, Gaspare Fossati (1809-1883), déjà célèbre à Istanbul et dont l'atelier est proche du sien ${ }^{4}$.

Peu à peu, au fil de ses collaborations, notamment avec le peintre alsacien CharlesGuillaume Hornig, mais surtout grâce à la société qu'il fonde, en 1856, avec l'architecte arménien Artin Serverian, Parvillée se fait connaître de la communauté pluri-culturelle istanbuliote et de la société ottomane. Ses travaux en Turquie, relayés par la presse et en particulier par le journal L'Illustration, sont connus des critiques français. Les projets s'enchaînent, commandes privées ou officielles de décors qu'il sculpte, restaure ou dont il dirige la réalisation, dans un style purement français ou dans le style ottoman: intérieurs de l'église Saint-Grégoire ou de la mosquée Ortaköy, du très à la mode "Café du Luxembourg » de Péra, du palais de Venise et des appartements du prince Abdülaziz au Palais de Dolmabahce 5 . En effet, depuis la fin des années 1830, contrainte de se moderniser face aux pays de l'ouest, la Turquie est en pleine réorganisation ; la période dite «des Tanzimat» (1839-1879) est marquée par de nombreuses réformes administratives, juridiques et militaires faites sur le modèle européen et dans les domaines public et privé, la mode "à la française», synonyme de modernité et d'élégance, connaît un vif succès. Quelques années plus tard en Europe, et en particulier en France, le mouvement «orientaliste» et le goût pour l'art turc connaissent un développement similaire, pour des raisons diamétralement opposées. Mais contrairement à la peinture qui élabore un vocabulaire iconographique propre à l'illustration du fantasme ou de l'intérêt que suscite "l'autre ", en architecture et dans les arts décoratifs la rencontre de ces deux cultures crée, de part et d'autre de la 
méditerranée, des œuvres souvent hybrides et étonnantes, faites de la juxtaposition d'éléments turcs ou français, ou "d'inspiration » turque ou française, plaqués sur des structures ainsi travesties ou réalisés selon les critères esthétiques de leur pays d'origine.

Lorsqu'en 1863, "Sergi-i Umum-i Osmaniye ", toute première exposition commerciale de l'Empire, est inaugurée à Sultanahmet, Léon Parvillée vit et travaille à Istanbul comme sculpteur et décorateur depuis douze ans. Il y a acquis la réputation d'un professionnel de goût et d'un homme érudit. Le gouvernement lui confie la création et la réalisation du décor de style ottoman du palais de l'exposition, palais néoclassique conçu par l'architecte français Antoine Bourgeois.

7 Quel rôle Parvillée a-t-il joué exactement dans la grande restauration de la ville de Brousse, ancienne capitale de l'Empire, qui démarre cette même année ${ }^{6}$ ? On sait qu'il est nommé, aux côtés de l'architecte italien Pietro Montani et de deux ingénieurs français, par le commissaire impérial Ahmed Vefik Effendi, pour expertiser les vestiges des monuments des $\mathrm{XIV}^{\mathrm{e}}$ et $\mathrm{XV}^{\mathrm{e}}$ siècles et restaurer certains d'entre eux. Le livre qu'il publie des années après son retour en France - Architecture et décoration turque au $X V^{e}$ siècle, publié par la maison Morel en 1874 - repose d'ailleurs essentiellement sur cette partie de son travail en Turquie. Selon ses propres notes, il conduit la « consolidation » de quatre mosquées (Ulu, Orhan et Yesil Cami, ainsi que la mosquée de Mourad II), du tombeau du sultan Mohammed I ${ }^{\text {er }}$ et d'un minaret.

Ce travail d'architecte se poursuit en 1867 pour l'Exposition universelle à Paris. Nommé " architecte de la commission impériale ottomane ", il remanie les plans et les décors d'abord conçus par l'architecte Barborini, de la porte triomphale et des trois pavillons de la section turque implantée sur le Champ de Mars: la mosquée, le kiosque du Bosphore et les bains. Ses dessins, ainsi que ceux du projet d'une chapelle mortuaire, sont exposés sous le promenoir couvert des jardins du Champ de Mars, dont un mur est réservé à la Turquie ${ }^{7}$. Pendant les travaux de restauration de Brousse, Léon Parvillée fait vraisemblablement plusieurs voyages entre la Turquie et la France. Revenu définitivement à Paris, il s'installe rue Malher comme « peintre en décors » en 1866.

9 Or, à l'Exposition universelle de 1867 précisément, il présente aussi, dans la section " céramique française ", ses premières réalisations en faïence de style islamique, qui le classent d'emblée parmi les céramistes et parmi les plus appréciés ${ }^{8}$. Ces réalisations prouvent qu'il s'est déjà beaucoup investi dans l'étude et le travail de cette matière. En 1884, il écrit :

Je me rappelle il y a vingt ans, [...] revenant de Brousse à Paris, M. Viollet-le-Duc m'encouragea vivement à étudier sur place les faïences des édifices d'Orient et à chercher les procédés de leur exécution'. Pris d'un bel entrain, j'achetais dans ce but tous les livres que je pus trouver qui traitaient de la matière [...]. Aucun ne m'indiquait la voie à suivre. [...] J'étais, je l'avoue, épuisé de lecture [...] et je n'y voyais pas plus clair. Ce fut un petit ouvrage, le plus modeste de tous, qui vint sérieusement à mon aide et m'ouvrit toute grande la route que je parcours aujourd'hui ${ }^{10}$.

Et ce qu'il expose en 1867, ce sont des carreaux de céramique architecturale en haut et bas relief. 


\section{Faire connaître l'art turc en France}

11 Cette même année, il achète un bâtiment au 15 quai de Bourbon à Saint-Maur-desFossés. Il y travaille jusqu'en 1870, d'abord en collaboration avec le sculpteur Alfred Salomon Flandrin, auquel s'associe, en 1868, le créateur et céramiste Amédée de Caranza $^{11}$, puis, en 1869 , avec Labbé ${ }^{12}$, qu'il a, comme Caranza, rencontré en Orient. Cette société est inscrite dans les almanachs du commerce comme fabrique de «briques émaillées pour la décoration du bâtiment genre oriental ». Après la destruction de sa fabrique, le céramiste, forcé de quitter Saint-Maur assiégée par les Prussiens, ouvre un nouvel atelier d' " architecture et décorations orientales » avec ses deux fils Achille et Louis, rue Neuve-Fontaine-saint-Georges à Paris en 1871, puis rue Fromentin en 1880. En 1882, la maison Parvillée, s'étant considérablement développée, fait construire de nouveaux locaux au 46 rue Caulaincourt dans le quartier de Montmartre.

12 À Paris comme à Istanbul, le céramiste travaille en collaboration avec de nombreux artistes et manufactures et noue un réseau de relations qui lui ouvre les portes des milieux artistiques et intellectuels français. Il a conservé des liens avec Viollet-le-Duc qui semble lui avoir confié la réalisation des décors des intérieurs de cheminée du château de Pierrefonds en 1866. Il travaille aussi avec plusieurs architectes dont Marcel Deslignières ou encore avec les peintres Émile Müller ou Boudier et ses réalisations pour les manufactures Champion, Lombard Fils, Gilardoni ou Huart de Longwy sont présentées au public lors de l'exposition de l'Union centrale de 1884 . Sa participation assidue aux expositions de l'Union centrale lui valent d'ailleurs de figurer parmi les céramistes représentés très tôt dans les collections du futur musée des arts décoratifs : le comité d'achats acquiert quatre de ses œuvres vers 1882, auxquelles s'ajoutent quatre autres données par le céramiste lui-même ou par le collectionneur charles Orange en 1900. Parvillée devient encore membre de l'Union céramique et chaufournière de France en 1879, avec laquelle il expose à plusieurs reprises. Citons encore, dans un tout autre registre, sa présence aux dîners des «Têtes de bois ", cercle d'artistes et d'intellectuels de tout bord, au café Procope, vers la fin des années $1870^{13}$.

L'essentiel de sa production reste, jusqu'à sa mort en 1885, la céramique architecturale - briques émaillées mais aussi panneaux décoratifs ou frises, ornés de rinceaux floraux et d'entrelacs géométriques dans le style turc d'abord, puis à décors historicistes ou éclectiques. Au début de l'année 1872, Léon Parvillée dépose d'ailleurs un brevet sur l'utilisation artistique ou industrielle de l'argile dans la décoration des habitations ou la réalisation d'ornements extérieurs comme les plaques de rues. Les commandes de grands ensembles décoratifs, intérieurs ou extérieurs, se succèdent à nouveau: décoration de l'hôtel de $\mathrm{M}^{\text {lle }}$ Mayer en 1873, de trois pièces de style ottoman dans l'appartement du comte Branicki en 1874 et, sans doute vers 1875, d'un salon de réception de style arabe dans l'hôtel particulier de Gaston Meunier, fils du chocolatier Émile-Justin Meunier; décoration d'un café rue Marengo en 1879 et du restaurant Champeaux place de la Bourse en $1882^{14}$ à Paris ; restauration du pavement du prieuré Sainte-Croix du château d'Eu et décor complet, japonisant, de l'établissement thermal de Bourbon-l'Archambault en $1882^{15}$ et encore décor de briques émaillées et de métopes sur les façades des lycées Racine à Paris en 1885 et Lakanal à Sceaux en 1884-1885 ${ }^{16}$. À ces commandes il faut ajouter les céramiques émaillées des façades du palais des expositions et du palais algérien, du pavillon de la ville de Paris, de la manufacture des tabacs et de l'Union céramique et chaufournière de France pour l'Exposition universelle 
de 1878 à Paris. Parvillée reçoit la médaille de la Société centrale des architectes pour la qualité de ces décors. Le "Grand Dépôt », grand magasin de faïence et de porcelaine, en copie les arabesques pour des panneaux de décors intérieurs « à l'orientale » vendus 65 francs le mètre.

Présent à chaque exposition de l'Union centrale des beaux-arts appliqués à l'industrie à partir de 1869 pour lesquelles il s'inscrit comme "architecte-décorateur ", il participe aussi à toutes les Expositions universelles entre 1867 et 1885 dans la section céramique d'art. Or dès le début des années 1870, Parvillée diversifie sa production et ajoute les arts décoratifs au catalogue de ses fabrications auxquels il applique les fruits de ses recherches sur la céramique architecturale. De multiples objets en faïence prennent peu à peu place aux côtés de ses panneaux et carreaux de terre cuite. À l'Exposition universelle de 1871 à Londres, il expose pour la première fois une paire de vases, des plats et une fontaine de style turc. Les encadrements de miroirs, plats, vases ou aiguières que Parvillée présente au public par la suite lui valent de nombreuses médailles et les éloges des critiques. Chaque pièce est d'ailleurs une œuvre à part entière, unique et essentiellement décorative (ill. 1).

III. 1 : Reproduction de la mosquée de Brousse exécutée par Léon Parvillée sur le Champs de Mars pour l'exposition universelle de 1867 à Paris

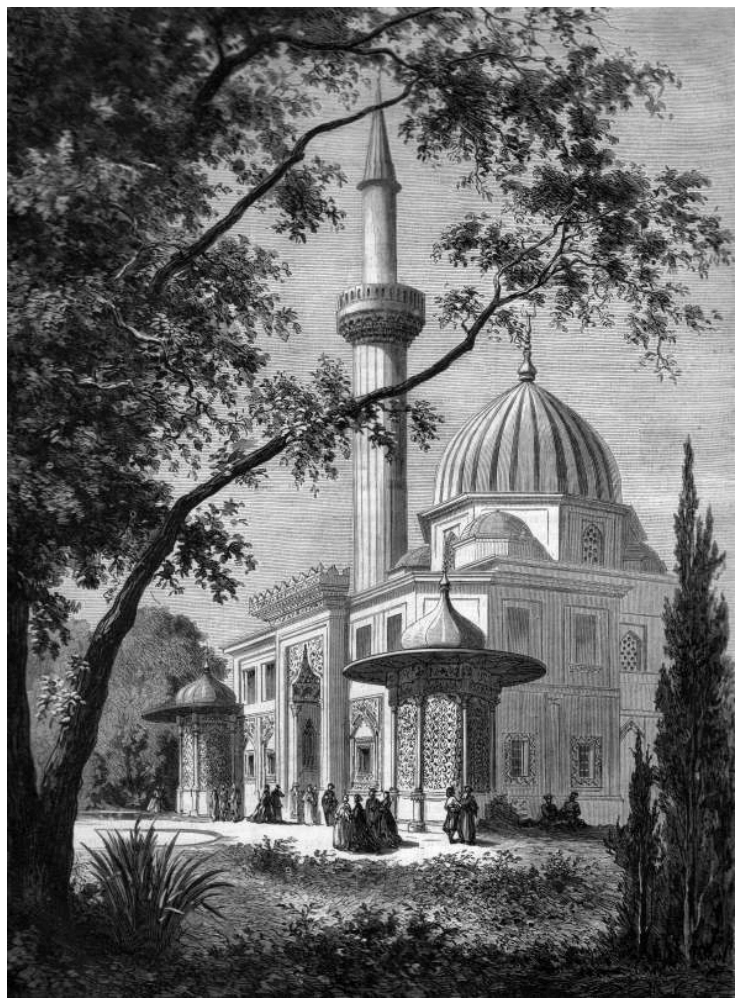

Dessin de Dieudonné Lancelot publié dans François Ducuing, L'Exposition universelle illustrée, éd. Dentu, 1867, t. 1, 480 p., p. $36,30,3 \times 21,5 \mathrm{~cm}$

Cl. Caroline Gronier. 


\title{
Les céramiques orientalistes de Parvillée : décors architecturaux et objets d'art
}

\author{
C'est l'architecture qui est la mère des arts \\ décoratifs [...]. \\ Le premier des décorateurs c'est l'architecte. ${ }^{17}$
} le cas du carrelage de la chapelle de la cathédrale de Langres ou le Plat des cigaliers réalisé pour Théodore Albanel en 1879. À l'art japonais nouvellement découvert, il emprunte des ornements naturalistes au dessin plus libre: les thermes de Bourbonl'Archambault en offrent le plus bel exemple. Ces styles s'ajoutent à l'inspiration orientale et se juxtaposent dans son œuvre plus qu'ils ne se succèdent, donnant à sa production le caractère éclectique qui marque tous les arts de la seconde moitié du XIX ${ }^{e}$ siècle. Le travail de ses cinq dernières années sera ensuite nettement naturaliste, mêlant fleurs et papillons dans des compositions asymétriques et excentrées. Pourtant, il faut reconnaître que, pendant plus de vingt ans, ses décors sont essentiellement inspirés par l'art turc.

\section{Les leçons de l'art ottoman}

Après une quinzaine d'années passées en Turquie, les architectures que Parvillée conçoit pour la section ottomane à Paris en 1867, l'estime que le gouvernement de l'Empire lui témoigne ainsi que la perfection des ornements islamiques qui ornent ses premières céramiques le placent d'emblée parmi les principaux spécialistes de l'Orient. Dès cette Exposition, il est perçu par ses compatriotes comme l'un des grands connaisseurs de l'art arabe, de son architecture comme de sa décoration.

Son livre, Architecture et décoration turque au XVe siècle, qui parait chez Morel en 1874, finit d'asseoir cette réputation. Viollet-le-Duc, qui en avait annoncé la parution dans son introduction à l'ouvrage de Jules Bourgoin, Les Arts arabes, paru l'année précédente ${ }^{18}$, en signe la préface. Associant le nom de Parvillée à celui de Bourgoin et de Duthoit, il place sur le même plan les recherches des trois hommes et les principes de géométrie élémentaire qu'ils déduisent, chacun, des arts islamiques.

D'autres artistes qui ont parcouru et étudié l'orient, M. Bourgoin [...], M. Duthoit, ont déjà montré que, pour le tracé des ornements sinon pour l'assemblage des tons, les procédés employés pour obtenir les compositions les plus compliquées et les plus harmonieuses en même temps, sont purement géométriques et élémentaires. [...] M. Parvillée, de son côté, en étudiant l'art auquel il applique la dénomination de turc, est arrivé à des résultats identiques. Or, pour que ces trois artistes consciencieux, travaillant en même temps, chacun de leur coté, tirent de leurs observations une même conséquence, il faut bien admettre qu'ils sont dans la vérité 19.

Désormais, Léon Parvillée est assimilé aux architectes rationalistes. Comme eux, il semble avoir appliqué les méthodes d'analyses rigoureuses de Viollet-le-Duc à son étude de l'art turc ; comme eux, il semble en tirer les leçons qui s'imposent pour mettre à la portée de tout créateur français « une explication raisonnée qui puissent servir de guide pour recomposer les ensembles de ce style, sans être obligé de copier servilement ceux qui existent $»^{20}$. 
si Parvillée démontre que les constructions turques reposent sur le triangle égyptien (triangle rectangle dont les côtés ont un rapport de 3-4-5), ce principe, il faut bien l'avouer, est la seule référence qu'il fasse à la géométrie. Il pose d'abord longuement les jalons historiques de la constitution de l'art ottoman - en douze pages sur seize pages de texte - et retrace encore chronologiquement la façon dont il a découvert, par hasard, l'application de la figure du triangle dans la coupe d'une ogive. La manière de tracer les ornements des monuments, puis ceux des objets, quelle que soit la matière dans laquelle ils sont faits, l'armature constituée par cette série de lignes, l'alternance des couleurs dont dépend l'harmonie de l'ensemble, et enfin la démonstration de ce fameux triangle, sont exposées plus que brièvement en à peine deux pages. De fait, Parvillée écrit dans son introduction :

Cette faible démonstration des principes que j'expose a reçu sur une vaste échelle toutes ses révélations dans le livre des Arts arabes, par J. Bourgoin ${ }^{21}$.

L'ouvrage est accompagné de cinquante chromolithographies pleine page pour la plupart: plans, coupes et vues des monuments restaurés à Brousse et des détails d'ornements en couleur. Peut-on donc réellement classer Parvillée parmi les théoriciens de l'art oriental ou son ouvrage se situe-t-il encore dans la lignée des recueils d'ornements ? Quoiqu'il en soit, le livre du céramiste, et de façon plus concrète, les architectures qu'il réalise pour l'Exposition de 1867, apportent au public, aux manufactures et aux fabricants, un témoignage vécu de l'art ottoman, jusque là encore bien méconnu.

\section{L'application de ces leçons dans l'œuvre de Parvillée}

21 L'Orient ne fournit pas aux arts décoratifs qu'un nouveau répertoire ornemental, il lui offre aussi, et même en premier lieu, un nouveau répertoire de formes. Après des œuvres de pure fantaisie dans lesquelles les habitants, la faune et la flore des pays arabes prêtent leur silhouette aux objets les plus divers, l'étude de l'architecture islamique donne aux dessinateurs des profils plus spécifiques. Minarets, coupoles, arcs outrepassés ou polylobés évoquent le charme du monde de l'Islam et donnent leur caractère à des œuvres monumentales françaises. Les fontaines exposées par Parvillée en 1871 ou par la manufacture Vieillard, exécutée sous la direction d'Amédée de Caranza en 1878, en sont des exemples significatifs : pour chacune d'entre elles, le fabricant a placé le bassin sous un arc brisé soutenu par de fines colonnettes, le fond de la niche ainsi formée étant encore découpé par un arc polylobé très évocateur. Bien sûr, les céramiques et les ferronneries arabes apportent aussi leurs galbes prononcés, leurs longs cols étroits et leurs formes fluides aux arts décoratifs. Les bouteilles dites persanes à panse généreuse par exemple sont très imitées. Le musée des arts décoratifs de Paris en possède de nombreux exemplaires réalisés par Eugène Collinot, Théodore Deck, Clément Massier ou Hippolyte Boulenger. Sèvres en présente aussi de nombreuses versions ornées de fleurs et de pâtes d'application aux Expositions. Il en va de même pour les aiguières sur piédouche et leur bassin aux formes élégantes dont le Victoria and Albert museum conserve une version de Parvillée particulièrement réussie, datant de 1875 .

Les ornements riches et complexes, très colorés pour la plupart, sont ensuite appliqués à la production artisanale puis industrielle: fleurs et feuillages, œillets d'Iznik, se déclinent en bouquets symétriques ou en compositions rayonnantes sur les petits

Livraisons de l'histoire de l'architecture, 17 | 2009 
objets les plus précieux jusqu'aux décorations architecturales. Les motifs géométriques en étoiles parent aussi une assiette présentée par Parvillée lors de l'exposition de l'Union centrale en 1874 et la façade du palais algérien qu'il crée pour l'Exposition universelle de 1878 .

Renouvelant les formes et les ornements, de même que les compositions traditionnelles, l'art arabe pousse encore les créateurs à développer leurs gammes chromatiques et se trouvera à la source du goût pour les reflets métalliques des faïences et des grès de la fin du siècle. Cherchant, dans cette optique, les moyens de rendre ses émaux plus résistants et plus lumineux, Léon Parvillée se rend à Murano en 1875 et met au point, à son retour, la peinture en couvertes colorées dont il pare et ses céramiques architecturales et ses objets. Le verre, coloré dans sa masse, est appliqué au pinceau par le peintre sur le biscuit sans engobe par couches successives, en fonction de l'intensité de ton souhaitée. Les couleurs, cuites ensuite au même grand feu, sont "inaltérables ", assure le fabriquant. Ce procédé, qui diffère des procédés décoratifs traditionnels sur faïence, précisément par sa composition sans plomb et son extrême translucidité après fusion est détaillé par Achille Parvillée dans le livre qu'il publie en 1884 chez Mary : Étude sur l'enseignement raisonné de l'art céramique ${ }^{22}$. Léon Parvillée est fier de préciser que la palette de couleurs développée sous sa direction est d'une richesse sans précédent. L'utilisation de cette technique est facile à distinguer car elle nécessite le tracé d'un fin cloisonnement entre les couleurs et donne un relief tout particulier à chaque zone colorée ${ }^{23}$. Ce cloisonnement fait d'ailleurs écho à l'armature des dessins ornementaux que Parvillée décrit dans son ouvrage, armature de plusieurs filets qui déterminent les zones à colorer. Cette précision et la netteté des détails feront le succès de Parvillée, et cette caractéristique fait de lui un artiste du XIXe siècle à part entière.

Si l'Orient apporte une source d'inspiration apparemment inépuisable dans les années 1860-1870, les céramistes s'attachent tous à obtenir des formes régulières, des détails d'une grande netteté ou des émaux sans bavure. Dans les décors impeccablement tracés, l'artisan ne laisse aucune trace de son travail. C'est cette opposition majeure entre les créations orientales et les réalisations occidentales dont témoigne la céramique de Léon Parvillée. Les fabricants ou les manufactures françaises ne copient pas l'Orient, ils en interprètent les formes, les décors, les techniques et produisent des œuvres qui correspondent aux goûts de leurs contemporains, à leur culture et qui mettent en évidence le niveau industriel de la France.

\section{NOTES}

1. L'« École royale gratuite de dessin, de mathématiques, de construction et de sculpture d'ornement en faveur des arts mécaniques ", puis « École nationale spéciale de dessin...» à partir de 1847, appelée communément la « Petite École » devient l'École nationale des arts décoratifs en 1877. Réorganisée dès le début du XIXe siècle afin de former des artisans qualifiés, l'enseignement y est diversifié sous la direction de Jean-Hilaire Belloc (1786-1866). Voir Renaud d'Enfert « De l'École royale gratuite de dessin à l'École nationale des arts décoratifs (1806-1877) », 
Histoire de l'École nationale supérieure des arts décoratifs (1766-1941), Journal de l'Ensad, no 24, novembre 2004, p. 64-107.

2. . Arch. nat., AJ 53, archives de l'École nationale des arts décoratifs de 1843 à 1848 .

3. . Remise au goût du jour au début du XIX ${ }^{\mathrm{e}}$ siècle, la technique de la «pâte de carton » imite la sculpture sur pierre. Résistants, légers et bon marché, les moulages de statues, d'objets ou d'ornements architecturaux fleurissent en France depuis les années 1830.

4. . Gaspare Fossati et son frère Giuseppe, architectes tessinois, sont chargés par le sultan Abdul Mecit de restaurer la basilique Sainte-Sophie en 1847.

5. Sur les travaux et les collaborations de Léon Parvillée à Istanbul voir Miyuki Aoki-Girardelli, "A Parisien in Istanbul : Léon Parvillée and His Notion of Turkish Art », International Congress of Turkich Art XIII, Budapest 2007, à paraître.

6. . Béatrice Saint-Laurent, «Léon Parvillée. His rôle as restorer of Bursa's monuments and his contribution to the exposition universelle of 1867 ", Hâmit Batu, Jean-Louis Bacquée-Grammont (dir.), L'Empire Ottoman, la République de Turquie et la France, éd. Isis, Istanbul-Paris, 1986, p. 247-282.

7. . Salaheddin Bey, La Turquie à l'Exposition universelle de 1867, Paris, Hachette, 1867, 256 p., p. 30 à 37 et p. 139.

8. . Contrairement à ce qu'écrira Victor de Luynes en 1871, Léon Parvillée ne participe pas à l'exposition des Beaux-arts appliqués à l'industrie organisée par le comité des inventeurs et des artistes industriels en 1863, au palais de l'industrie. Son nom ne figure dans aucun des catalogues de l'époque. - V. de Luynes, "poteries décoratives ", Exposition internationale de Londres de 1871, France, Commission supérieure, rapport, classe III, Paris, impr. Clayes, 1872, p. 118 à 131.

9. . Léon Parvillée rentre en effet à Paris avec de nombreux croquis et dessins réalisés d'après les monuments vus en Turquie, mais également avec une collection de fragments de décors et d'objets acquis sur place.

10. . Léon Parvillée, introduction à Achille Parvillée, Étude sur l'enseignement raisonné de l'art céramique, Paris, éd. Mary et fils, 1884, 60 p., p. 3.

11. . Amédée de Caranza, que l'on sait né à Istanbul, entre en 1872 dans l'atelier de décoration de la faïencerie de Longwy où il développe la technique des émaux cloisonnés qui feront sa réputation et la gloire de la faïencerie. Il travaille ensuite, dans les années 1880 , pour la manufacture Vieillart à Bordeaux.

12. . À l'Exposition universelle de 1867, deux toiles de «Labbé » sont exposées avec les dessins de Léon Parvillée dans la section turque. Peut-être s'agit-il du peintre orientaliste Émile Charles Labbé (1820-1885) qui, d'abord peintre paysagiste, se tourne vers des sujets orientalistes dans les années 1840. E. C. Labbé fait notamment deux voyages en Algérie avec Eugène Fromentin et Armand du Mesnil. Il part ensuite pour la Grèce et vit quelque temps à Istanbul où il travaille vraisemblablement pour le sultan, participant à une expédition dans les couvents du mont Athos pour le gouvernement ottoman aux côtés du photographe Ernest de Caranza. Revenu en France vers 1860 , il prend la direction de la nouvelle École des beaux-arts d'Alger en 1881.

13. . Dans la préface de l'ouvrage Le Livre des têtes de bois de Georges Courteline (pseudonyme de Georges Moinaux, membre de l'Académie Goncourt en 1928), publié chez Charpentier en 1883, Saint-Juirs (ou René Delorme, romancier) dresse la liste des membres de ce cercle de peintres, sculpteurs, céramistes, musiciens et gens de lettres dont fit partie, entre autres, Guy de Maupassant.

14. . Le restaurant Champeaux, dont le chef Catelain se fit connaître pour sa fameuse recette du "poulet champeaux » dans les années 1840 , est très à la mode dans la seconde moitié du siècle notamment grâce à son jardin d'hiver. Il est détruit par une explosion le 20 novembre 1898.

15. . Le décor de l'établissement thermal de Bourbon-l'Archambault est conservé in situ dans un état irréprochable, constituant le plus grand ensemble céramique de la maison Parvillée connu et 
conservé à ce jour. Le bâtiment est restauré entre 1881 et 1891 par l'architecte Charles Le Cœur, les panneaux ornementaux sont livrés par les frères Parvillée entre 1891 et 1896.

16. . Pour plus de détails sur les travaux de la fabrique Parvillée voir Caroline Gronier, Léon Parvillée, céramiste, architecte-décorateur (1830-1885), mémoire de D.E.A. en histoire de l'art contemporain, Paris IV, 1996 et Elsa Schneider-Manuch, «Léon Parvillée (1830-1885), parcours d'un pionnier de la céramique architecturale ", Recherche en Histoire de l'art, $\mathrm{n}^{\circ} 1$, ClermontFerrand, 2002, p. 115 à 136.

17. . César Daly, «L'École impériale de dessin », Revue de l'Architecture et des travaux publics, 1863, Paris, vol. XX, col. 158.

18. . Eugène Viollet-le-Duc, préface à Jules Bourgoin, Les Arts arabes, Paris, V ${ }^{\mathrm{ve}}$ A. Morel et $\mathrm{C}^{\mathrm{ie}}$, 1873,36 p. et $91 \mathrm{pl}$.

19. . Eugène Viollet-le-Duc, préface à Léon Parvillée, Architecture et décoration turque au $X V^{e}$ siècle, Paris, $V^{\mathrm{ve}}$ A. Morel et $\mathrm{C}^{\mathrm{ie}}, 1874,16 \mathrm{p}$. et $50 \mathrm{pl}$.

20. . Léon Parvillée, Architecture et décoration turque au XVe siècle, ibid., introduction.

21. . Léon Parvillée, Architecture et décoration turque au XVe siècle, ibid., introduction.

22. . Achille Parvillée Étude sur l'enseignement raisonné de l'art céramique, op. cit.

23. Si Eugène Collinot cerne ses motifs d'un trait fin en noir ou en couleur - genre décoratif pour lequel il dépose un brevet en 1864 - les couvertes en verre coloré et la technique du cloisonné apparaissent pour la première fois dans l'œuvre de Théodore Deck en 1874. Les procédés utilisés par Parvillée puis par la manufacture Vieillard à Bordeaux et par Caranza à Longwy seront similaires. Comme beaucoup de procédés, les verres colorés sont aussitôt imités, mais leur fabrication est délicate. Certaines copies, qui font entrer le plomb dans leur composition, deviennent troubles et grises à la lumière artificielle.

\section{RÉSUMÉS}

Élève de Viollet-le-Duc, sculpteur, décorateur, chargé par le gouvernement ottoman de la restauration de la ville de Brousse puis des installations turques de l'exposition universelle de 1867, céramiste orientaliste reconnu et maintes fois médaillé, créateur du magnifique décor japonisant de l'établissement thermal de Bourbon-l'Archambault, défenseur de l'architecture polychrome, chercheur et historien de l'art, Léon Parvillée (1830-1885) est un artiste "éclectique » dans tous les sens du terme. Sa passion pour la céramique, née de ses travaux d'ornemaniste puis d'architecte, le pousse sans cesse à faire passer ses recherches techniques ou esthétiques de l'objet au bâtiment ou du bâtiment à l'objet. Puisant dans tous les domaines et dans toutes les références, les décors et les émaux qu'il compose nouent un dialogue permanent entre architecture et arts décoratifs.

Student of Viollet-le-Duc, sculptor, decorator, Léon Parvillée is mandated by the Ottoman government both for the restoration of the city of Bursa and for the turkish installations at the World's Fair of 1867 in Paris. Renowned and many times medal winner as an orientalist ceramist, creator of the splendid decoration of the thermal baths of Bourbon-l'Archambault in a Japaneseinspired style, defender of polychrome architecture, researcher and historian of art, Léon Parvillée (1830-1885) is indeed a real « eclectic» artist. His passion for ceramic, born from his work of ornemanist and architect, pushes him in an ongoing quest to link object and building through his technical and aesthetic researches. Drawing from all fields and from all references, 
the decorations and the enamels he composes tie a permanent dialogue between architecture and decorative arts.

Der Schüler Viollet-le-Ducs, Bildhauer und Ornamentist Léon Parvillée (1830-1885) wurde von der ottomanischen Regierung beauftragt, zuerst die Stadt Bursa zu restaurieren, dann die türkischen Einrichtungen der Weltaustellung $1867 \mathrm{zu}$ errichten. Er wurde als orientalister Keramiker gerühmt und so mehrmals mit Medaillen ausgezeichnet, aber er schuf auch das wertvolle Dekor im japonischen Stil des Thermenbades von Bourbon-l'Archambault. Léon Parvillées Forschungen im Gebiet der Kunstgeschichte sowie dessen Neigung zur Polychromie in der Architektur lassen ihn als Eklektistist in jedem Sinn des Begriffs bezeichnen. In seinen Werken als Ornamentist und als Architekt bevorzugt er Keramik, so dass er in diesem Gebiet immer wieder neue technische und esthetischen Forschungen unternahm. Die Modelle seiner Ornamente und Emaillen schöpfte er aus verschiedenen Bereichen, so dass ein stetiger Dialog zwischen Architektur und dekorativer Kunst zustandekommt.

\section{AUTEUR}

\section{CAROLINE GRONIER}

Caroline Gronier, née en 1970, est titulaire d'une maîtrise d'histoire de l'art sur L'influence de l'art islamique sur les arts décoratifs européens entre 1867 et 1878 et d'un DEA consacré à Léon Parvillée, céramiste, architecte-décorateur (1830-1885), obtenus à l'université de Paris IVSorbonne. Après avoir étudié les collections de céramiques du musée des arts décoratifs de Paris, elle a soutenu sa thèse de doctorat sur La faïence et le grès d'art en France pendant la seconde moitié du XIX ${ }^{e}$ siècle sous la direction de Bruno Foucart et d'Odile Nouvel en avril 2002. Après avoir obtenu le diplôme supérieur en techniques documentaires de l'INTD, elle a exercé comme documentaliste iconographe pendant plusieurs années. Elle mène actuellement des recherches sur la céramique orientaliste française de la seconde moitié du XIX ${ }^{\mathrm{e}}$ siècle. Adresse électronique : caroline.baillart@free.fr. 